\title{
Anatomy of plasma turbulence
}

\author{
TAKUMA YAMADA ${ }^{1 *}$, SANAE-I. ITOH ${ }^{1}$, TAKASHI MARUTA ${ }^{2}$, NAOHIRO KASUYA ${ }^{3}$, YOSHIHIKO NAGASHIMA ${ }^{1}$, \\ SHUNJIRO SHINOHARA ${ }^{2}$, KENICHIRO TERASAKA ${ }^{2}$, MASATOSHI YAGI ${ }^{1}$, SHIGERU INAGAKI ${ }^{1}$, \\ YOSHINOBU KAWAI ${ }^{1}$, AKIHIDE FUJISAWA ${ }^{3}$ AND KIMITAKA ITOH ${ }^{3}$
}

\author{
${ }^{1}$ Research Institute for Applied Mechanics, Kyushu University, 6-1 Kasuga-koen, Kasuga, Fukuoka 816-8580, Japan \\ ${ }^{2}$ Interdisciplinary Graduate School of Engineering Sciences, Kyushu University, 6-1 Kasuga-koen, Kasuga, Fukuoka 816-8580, Japan \\ ${ }^{3}$ National Institute for Fusion Science, 322-6 Oroshi-cho, Toki, Gifu 509-5292, Japan \\ *e-mail: takuma@riam.kyushu-u.ac.jp
}

Turbulence is a state of fluids and plasma where nonlinear interactions including cascades to finer scales take place to generate chaotic structure and dynamics ${ }^{1}$. However, turbulence could generate global structures ${ }^{2}$, such as dynamo magnetic field, zonal flows ${ }^{3}$, transport barriers, enhanced transport and quenching transport. Therefore, in turbulence, multiscale phenomena coevolve in space and time, and the character of plasma turbulence has been investigated in the laboratory $y^{4-10}$ as a modern and historical scientific mystery. Here, we report anatomical features of the plasma turbulence in the wavenumber-frequency domain by using nonlinear spectral analysis including the bi-spectrum ${ }^{11}$. First, the formation of the plasma turbulence can be regarded as a result of nonlinear interaction of a small number of irreducible parent modes that satisfy the linear dispersion relation. Second, the highlighted finding here, is the first identification of a streamer (state of bunching of drift waves ${ }^{12,13}$ ) that should degrade the quality of plasmas for magnetic confinement fusion ${ }^{14,15}$. The streamer is a poloidally localized, radially elongated global structure that lives longer than the characteristic turbulence correlation time, and our results reveal that the streamer is produced as the result of the nonlinear condensation, or nonlinear phase locking of the major triplet modes.

Fluctuation measurements were carried out on the Large Mirror Device-Upgrade linear plasma device ${ }^{16}$ (Fig. 1). The axial length of the vacuum vessel is $z=3.74 \mathrm{~m}$ and the cylindrical plasma is confined by an axial magnetic field of $0.09 \mathrm{~T}$. ( $x$ : horizontal, $y$ : vertical, $z$ : axial, $r$ : radial and $\theta$ : poloidal direction.) Positive and negative poloidal directions correspond to the electron and ion diamagnetic drift directions, respectively. The plasma is generated by a helicon wave (the radiofrequency $(7 \mathrm{MHz})$ power is $3 \mathrm{~kW}$, excited by a double-loop antenna around a quartz tube with an axial length of $0.4 \mathrm{~m}$ and an inner diameter of $9.5 \mathrm{~cm}$ ). The quartz tube is filled with argon gas with a pressure of $0.2-0.8 \mathrm{~Pa}$. A linear plasma (radius of $5 \mathrm{~cm}$, electron density/temperature of $10^{19} \mathrm{~m}^{-3} / 3 \mathrm{eV}$ ) is generated inside the vacuum vessel ${ }^{16}$. A 64 -channel poloidal probe array is installed at the plasma radius $r=r_{\mathrm{p}}=4 \mathrm{~cm}$ (where the density gradient is steep) and axial position $z=1.885 \mathrm{~m}$. A 48 -channel radially movable probe array ${ }^{17}$ is installed at the axial position $z=1.625 \mathrm{~m}$. (All 48 channels are used for measurement at $r \geq r_{\mathrm{p}}$, and 24 channels are used at $r<r_{\mathrm{p}}$, such as $r=2 \mathrm{~cm}$.) A two-dimensionally (2D) movable probe, which is movable in the $x-y$ plane in the plasma cross-section, is installed at the

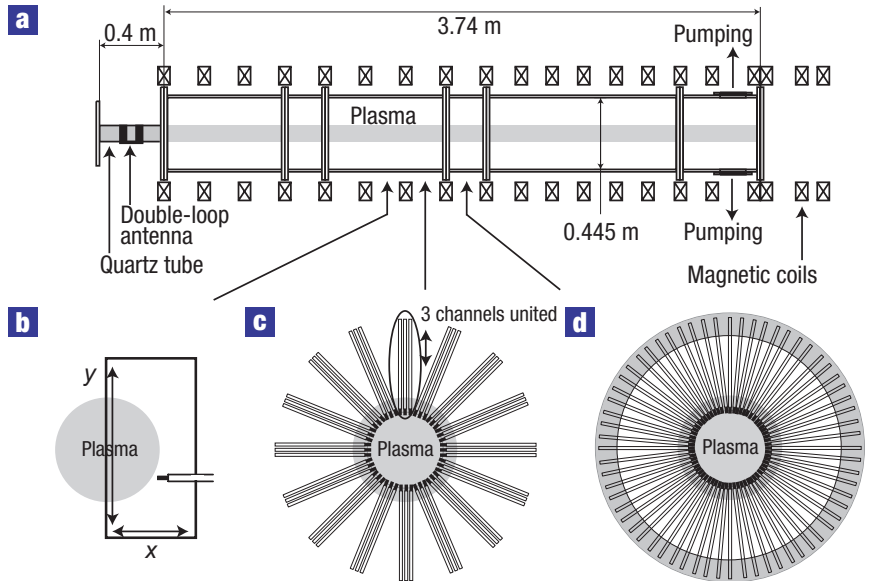

Figure 1 Schematic view of the plasma device and probes. a, Large Mirror Device-Upgrade linear plasma. b, 2D probe. c, 48-channel radially movable probe array. d, 64-channel poloidal probe array. One channel of the 48-channel probe array is used as a reference for the $2 \mathrm{D}$ probe.

axial position $z=1.375 \mathrm{~m}$. One of the 48 -channel probes at the $(x, y)=\left(r_{\mathrm{p}}, 0\right)$ position is used as a reference probe for the $2 \mathrm{D}$ probe. With these three systems of probes, the quasi-2D nature of turbulence is studied.

We first give an over-all description of fluctuation dynamics. Owing to the steep radial density gradient, drift-wave fluctuations (which originate from resistive drift-wave instabilities ${ }^{1}$ ) are excited. Figure $2 \mathrm{a}$ shows the spatiotemporal behaviour of the ion saturation-current fluctuation (inferred as a density fluctuation) measured with the 64-channel probe array. The argon pressure of this discharge was $0.27 \mathrm{~Pa}$. The spatiotemporal behaviour is in a chaotic regime, that is, the two-time and two-point correlation measurement shows that the correlation time is within a few oscillation periods (a few hundred microseconds) and the poloidal correlation length is much shorter than the poloidal arc length $2 \pi r_{\mathrm{p}}$.

By 2D Fourier transformation (poloidal angle $\theta$ to poloidal mode number $m$ and time $t$ to frequency $f$ ), the fluctuation 

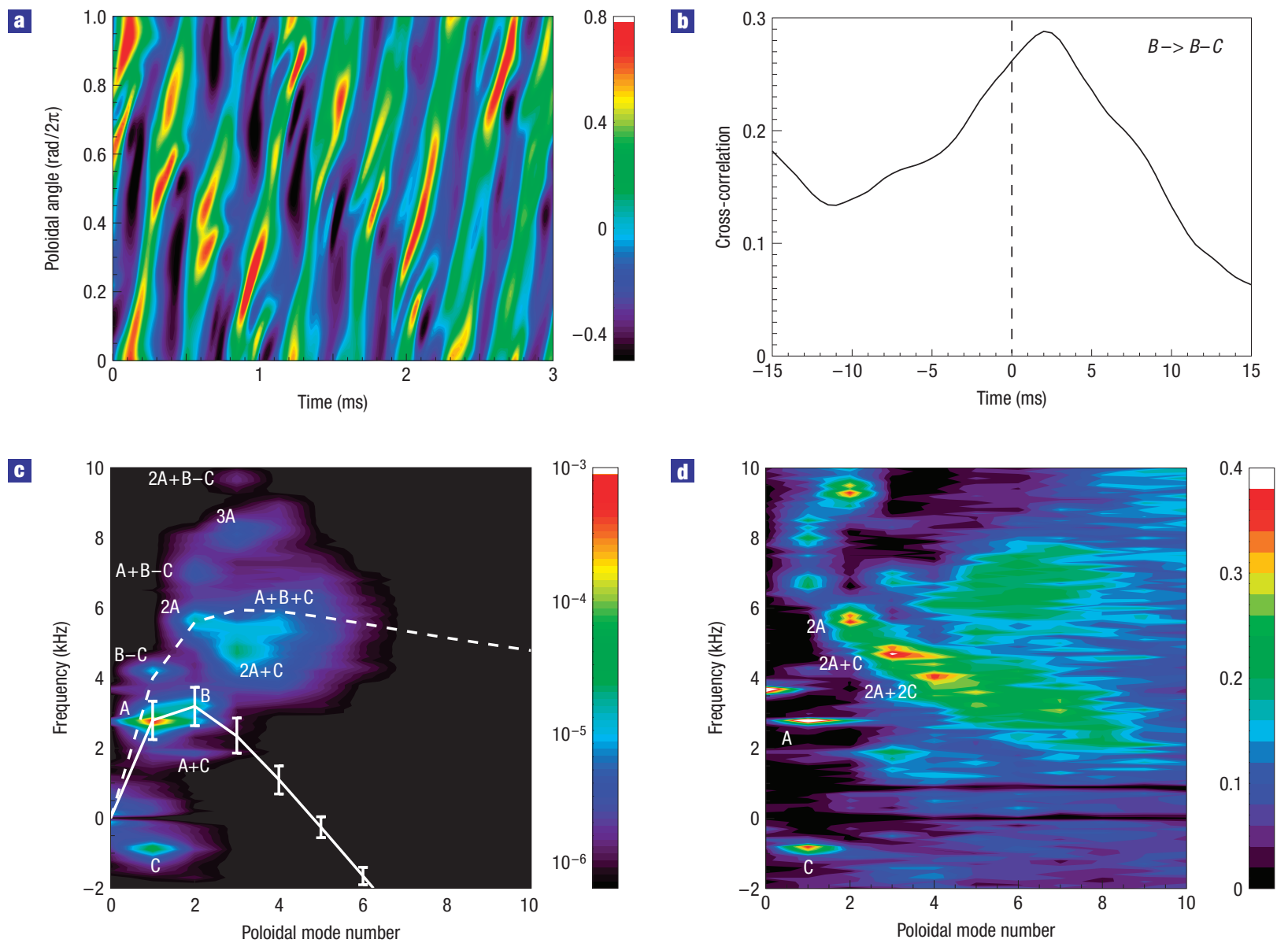

Figure 2 Spectral and bi-spectral analyses of fluctuations. a, Spatiotemporal behaviour of the ion saturation-current fluctuation, measured with the 64-channel poloidal probe array (arbitrary units, contour). The positive direction of the vertical axis corresponds to the electron diamagnetic direction. Bunching of the fluctuation is propagating in the ion diamagnetic direction. b. Cross-correlation function of the spectral components B and B-C. The horizontal axis indicates time delay of B-C from B. c, Power spectrum $S(m, f)$ (arbitrary unit, contour) over plotted with the linear dispersion relation (solid line) calculated by a numerical simulation code. Error bars are standard deviations of several results using possible electron density profiles. The dashed line indicates the linear dispersion where the mean $E \times B$ drift motion is neglected. The calculated eigenmodes have positive growth rates. $\mathbf{d}$, Bi-coherence analysis for triplets among excited fluctuations and the peak $\mathrm{C}$; contour of squared bi-coherence is shown.

is decomposed into a number of fluctuation modes. Figure $2 \mathrm{c}$ shows the $2 \mathrm{D}$ power spectrum $S(m, f)$. Positive frequency indicates propagation in the electron diamagnetic direction in the laboratory frame. The component propagating in the electron diamagnetic direction seen in Fig. $2 \mathrm{a}$ is the one at $(m, f)=(1,2.8 \mathrm{kHz})$, which is marked A in Fig. 2c. In the figure, the linear dispersion relation calculated by a numerical simulation code ${ }^{18}$ is also plotted with a white line. (A uniform rotation by a d.c. radial electric field is imposed, the magnitude of which is set by adjusting the $m=1$ peak to the eigenfrequency. This electric field is within an error-bar of the profile measurement.) The fluctuation modes $\mathrm{A}$ and $\mathrm{B}[(m, f)=(2,3.2 \mathrm{kHz})]$ satisfy the linear dispersion relation of drift waves, whereas other peaks are away from the dispersion relation. The peak at $(m, f)=(1,-0.9 \mathrm{kHz})$, which is considered to be a (marginally stable) flute mode, is labelled as $\mathrm{C}$ and all of the other peaks appearing in Fig. $2 \mathrm{c}$ can be expressed by addition and/or subtraction of $\mathrm{A}, \mathrm{B}$ and $\mathrm{C}$. Each peak is expressed as $\mathrm{A}+\mathrm{C}, \mathrm{B}-\mathrm{C}, 2 \mathrm{~A}, 2 \mathrm{~A}+\mathrm{C}, \mathrm{A}+\mathrm{B}-\mathrm{C}, 3 \mathrm{~A}$, $2 \mathrm{~A}+\mathrm{B}-\mathrm{C}, \mathrm{A}+\mathrm{B}+\mathrm{C}$ and so on. These peaks are considered to be quasimodes, which are driven by nonlinear couplings of the primary modes.
The mode couplings are confirmed by bi-spectral analysis ${ }^{11}$, which indicates the degree of nonlinear coupling between three modes (1, 2 and 3 ) satisfying the matching condition $\left(m_{1}+m_{2}=m_{3}\right.$ and $\left.f_{1}+f_{2}=f_{3}\right)$. (For accurate bi-spectral analysis, multiprobe measurement with fine positioning is realized ${ }^{19}$.) Carrying out bi-spectral analysis (combinations up to $m=5$ with frequencies $f_{1}$ and $f_{2}$ being scanned from -10 to $10 \mathrm{kHz}$ ), the quasimodes are found to be created sequentially by nonlinear coupling processes from $\mathrm{A}, \mathrm{B}$ and $\mathrm{C}$ in Fig. 2c. The amplitude correlation technique ${ }^{20,21}$ supports our idea that $\mathrm{A}, \mathrm{B}$ and $\mathrm{C}$ are the parent modes. Figure $2 \mathrm{~b}$ shows an example. It shows the cross-correlation function of the envelopes of B and B-C. The peak of the cross-correlation function is seen at $+2 \mathrm{~ms}$, which indicates that $\mathrm{B}-\mathrm{C}$ responds $2 \mathrm{~ms}$ after the variation of $\mathrm{B}$. (This delay is consistent with the autocorrelation time $\sim 3 \mathrm{~ms}$ of $\mathrm{B}-\mathrm{C}$, which is evaluated from the half-width of the peak of the spectrum.) From this fact, we can estimate that B is a parent of B-C. In this way, we determined that $\mathrm{A}, \mathrm{B}$ and $\mathrm{C}$ are the parent modes. In addition to the peaks of quasimodes, broadband components appear in the $(m, f)$ spectra, indicating fluctuations with very short lifetimes. They are attributed to granulations in fluctuations ${ }^{22}$. Thus, we have 
identified three irreducible elements of complex plasma turbulence. This number of irreducible components is found to be close to the correlation dimension calculated in a certain timescale.

The successive generations of $2 \mathrm{~A}, 3 \mathrm{~A}, \mathrm{~A}+\mathrm{B}+\mathrm{C}$ and so on show the cascade of energy to smaller-scale perturbations. In addition to cascades, theories have predicted a streamer structure, that is, a bunching (self-focusing) of fluctuations in the direction of wave propagation ( $\theta$-direction) with a small radial wavenumber. The streamer in this letter is the state of fluctuations where the intensity of the carrier wave is modulated in the direction of wave propagation by nonlinear interaction (self-focusing), whereas the envelope in the radial direction is less modified ${ }^{3,12-15}$. Figure 2a shows the emergence of the streamer. Thin stripes denote waves, and their envelope is modulated in the $\theta$-direction. The peaks (and troughs) of the envelope propagate slowly in the ion diamagnetic direction. The timescale of propagation of the envelope (in the laboratory frame), which is observed to be a few milliseconds, is about a few to ten times larger than that of waves. Taking into account the plasma rotation, which is introduced in the analysis of wave dispersion, reduces the rotation frequency of the envelope in the plasma frame to $\mathrm{O}(100 \mathrm{~Hz})$, and enhances the timescale separation between the waves and the envelope. The peak of the envelope propagates in the ion diamagnetic direction with $f \sim-0.9 \mathrm{kHz}$, which is the same as $\mathrm{C}$ $[(m, f)=(1,-0.9 \mathrm{kHz})]$. This propagation is created by nonlinear coupling of many fluctuation components with $m \geq 2$, such as $\mathrm{X}$ and $\mathrm{X} \pm \mathrm{C}$. The peak $\mathrm{X}$ is, for example, $2 \mathrm{~A}, \mathrm{~A}+\mathrm{B}$ and so on. The coexistence of multiple triplets (each of which indicates a poloidally focused wave component) is confirmed by the bi-spectral analysis. Figure $2 \mathrm{~d}$ shows the squared bi-coherence between $\mathrm{X}, \mathrm{C}$ and X+C (horizontal axis: $m_{1}$ of X, vertical axis: $f_{1}$ of $\mathrm{X}$ and $m_{2}=1$, $\left.f_{2}=-0.9 \mathrm{kHz}, m_{3}=m_{1}+1, f_{3}=f_{1}-0.9 \mathrm{kHz}\right)$. The nonlinear coupling of $\mathrm{C}$ with other quasimodes with $m \geq 2$ (such as $2 \mathrm{~A}$ ) is evident. In addition, the bi-phase is almost the same for any peaks of $\mathrm{X}$ and is slightly higher than 0 . This corresponds to the fact that the large-amplitude region appears just before the maximum of $\mathrm{C}$.

Now we focus on the analysis of the streamer by considering another discharge with more obvious streamer existence, to show explicitly that the streamer (nonlinear amplitude bunching in poloidal direction) has long radial extension and that the phase locking in the nonlinear interaction holds for a very long time compared with the correlation time of fluctuations $(\mathrm{O}(100 \mu \mathrm{s}))$. For clarity, we illustrate possible streamer structure in linear magnetized plasma in Fig. 3a based on nonlinear simulation (details of the simulation are described in ref. 18). The drift waves (thin stripes propagating in the positive $\theta$-direction) and the amplitude modulation are seen. The modulation envelope slowly rotates in the negative $\theta$-direction. The streamer structure was shown to be extended in the radial direction by two poloidal probe arrays in experiments. Figure $3 \mathrm{~b}-\mathrm{d}$ shows the result of simultaneous measurements of the ion saturation-current fluctuations $I_{\text {is }}$ by two poloidal probe arrays at different $r$ (and $z$ ) positions. The argon pressure was decreased to $0.20 \mathrm{~Pa}$ to obtain a stronger and longer-lasting streamer structure. (No fluctuation components of B origin appeared in this case.) Figure $3 \mathrm{c}$ shows the spatiotemporal behaviour of $I_{\text {is }}$ measured with the 64-channel poloidal probe array $(r=4 \mathrm{~cm}$ and $z=1.885 \mathrm{~m})$. Figure $3 \mathrm{~d}$ shows the temporal behaviour at $\theta=0$. The bunching of the fluctuations is clear in Fig. 3b,c. Figure 3b shows the spatiotemporal behaviour of the $I_{\text {is }}$ measured with the 24-channel poloidal probe array $(r=2 \mathrm{~cm}$ and $z=1.625 \mathrm{~m})$ for the same discharge as in Fig. 3c. Signals from two arrays of probes show very high coherence (over 0.9 for $\delta z=0.26 \mathrm{~m}$ ) and the axial wavelengths of drift waves are much longer than $\delta z$ (axial mode number $n \leq 3$ ). The self-focusing region, where $m \geq 2$ components are strongly
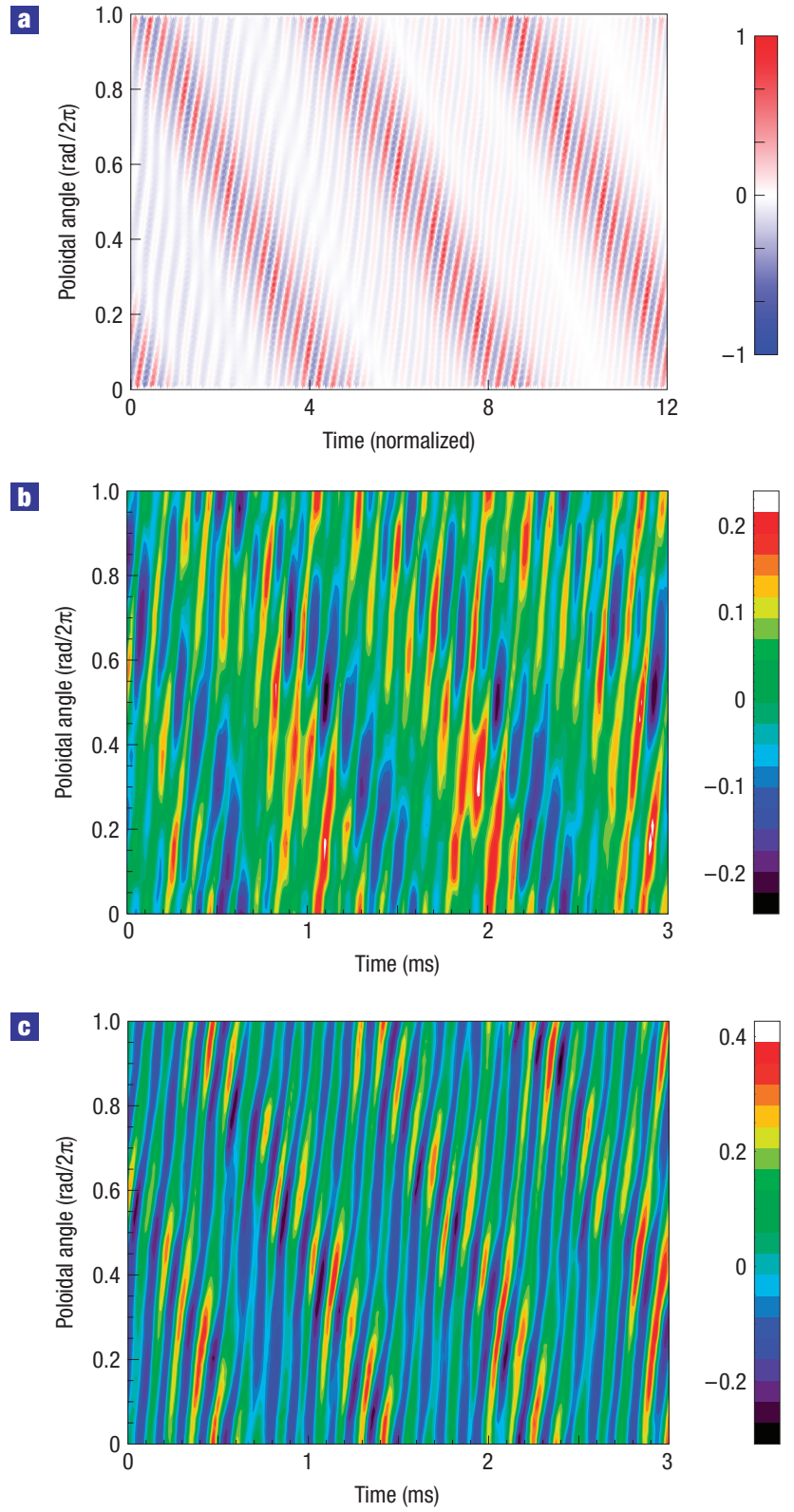

d

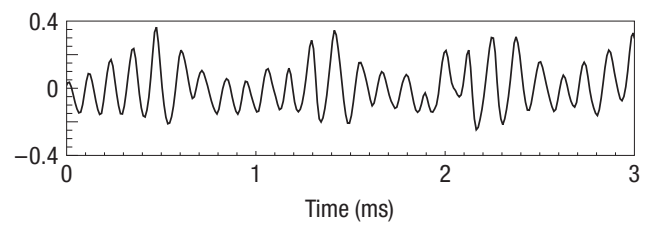

Figure 3 Time evolution of streamer structures. a, Obtained from a numerical simulation of drift waves in linear magnetized plasma. Density fluctuations (normalized by the maximum amplitude) are shown, where the time is normalized by the drift-wave oscillation period. The fluctuations are observed on a rotating frame with the rotation frequency of 0.3 . The simulation parameters are similar to the experimental conditions, but are not identical. For instance, the profile of the mean plasma density is modelled with a simple smooth function, the poloidal mode numbers of the carrier waves are $m=4$ and 5 and the temperature is assumed to be constant. b,c, Simultaneous measurement of the spatiotemporal behaviours of the ion saturation-current fluctuations by 24 channels, $r=2 \mathrm{~cm}$ and

$z=1.625 \mathrm{~m}$ (b) and 64 channels, $r=4 \mathrm{~cm}$ and $z=1.885 \mathrm{~m}$ (c). The poloidal mode numbers of the carrier waves are mainly $m=2$ and 3 . $\mathbf{d}$, Temporal behaviour of $\mathbf{c}$ at $\theta=0$. 

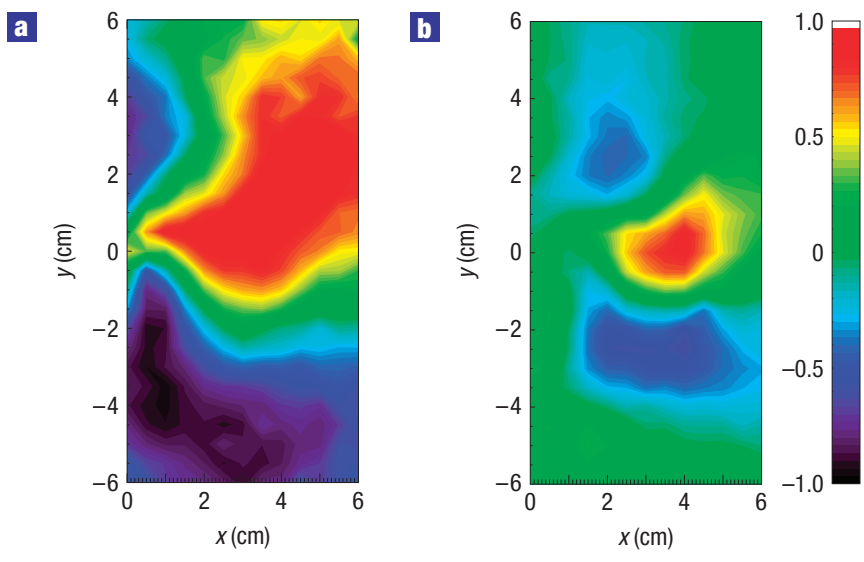

G

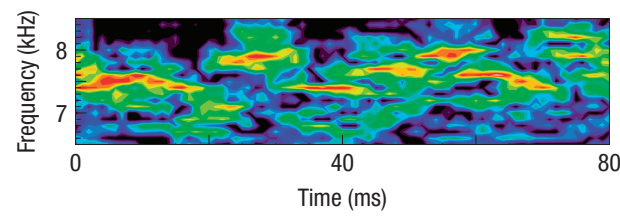

$10^{-4}$

d

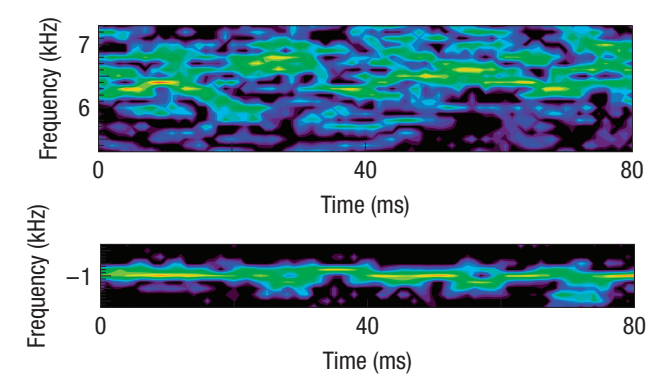

$10^{-5}$

e

Figure 4 Features of the streamer structure. $\mathbf{a}, \mathbf{b}$, Real parts of cross-spectra between the reference probe $[(x, y, z)=(4 \mathrm{~cm}, 0,1.625 \mathrm{~m})]$ and the $2 \mathrm{D}$ movable probe $(z=1.375 \mathrm{~m})$. Frequency ranges are $7.8 \mathrm{kHz}$ (one of the main components of the streamer) in a and $10-15 \mathrm{kHz}$ (broadband region) in b. c-e, Time developments of $m=2$ (c), $m=3$ (d) and $m=1$ (e) components calculated by short-time Fourier transformation.

fluctuating, agrees in the two figures. Therefore, the streamer is shown to have a long structure (comparable to the device size) in the radial direction.

Further direct evidence of the radial extension of the streamer structure was confirmed by the $2 \mathrm{D}$ probe. The streamer structure in Fig. 3 is generated mainly by $2 \mathrm{~A}[(m, f)=(2,7.8 \mathrm{kHz})]$, $2 \mathrm{~A}+\mathrm{C}[(m, f)=(3,6.6 \mathrm{kHz})]$ and broadband components up to $[(m, f) \sim(6,15 \mathrm{kHz})]$. Figure $4 \mathrm{a}, \mathrm{b}$ shows real parts of cross-spectra between the $2 \mathrm{D}$ probe $(z=1.375 \mathrm{~m})$ and the reference probe $[(x, y, z)=(4 \mathrm{~cm}, 0,1.625 \mathrm{~m})]$ with frequency components of $2 \mathrm{~A}$ $(7.8 \mathrm{kHz})$ and broadband components $(10-15 \mathrm{kHz})$, respectively. As each component extends radially, the streamer must have a radially extended structure. The phase structure is long in the radial direction with some spiral components. These spiral components are considered to be induced by the d.c. radial electric field.

The nonlinear lock between two neighbouring poloidal mode numbers is confirmed up to $m=6$, and several triplet pairs are found to coexist. It is striking that, although a one-point correlation for a high-pass signal $(f>2.5 \mathrm{kHz}$, which is a characteristic scale for fluctuations) gives a short correlation time (a few hundred microseconds), the nonlinear interaction between each triplet pair survives longer than $10 \mathrm{~ms}$, showing long-lasting (meso-scale) nonlinear structures in the turbulent plasma. More precisely, even though the central frequency of higher modes $\mathrm{X}$ varies in time (on a timescale of $10 \mathrm{~ms}$ ), the coupling between $\mathrm{X}, \mathrm{X} \pm \mathrm{C}$ and $\mathrm{C}$ is preserved. An example is shown in Fig. 4. Figure 4c-e shows time developments of $m=2,3$ and 1 components, which correspond to $2 \mathrm{~A}, 2 \mathrm{~A}+\mathrm{C}$ and $\mathrm{C}$, respectively. Although the frequency of $2 \mathrm{~A}$ varies in time, the frequency difference between $2 \mathrm{~A}$ and $2 \mathrm{~A}+\mathrm{C}$ preserves and corresponds to the steady frequency of $\mathrm{C}$, as is clearly demonstrated in Fig. 4c-e.

\section{METHODS}

\section{SPECTRAL ANALYSIS}

When there are $M$ probe tips in the poloidal direction with equal space, the poloidal angle $\theta$ equals $2 \pi i / M$, where $i$ is an integer. When the time evolution is obtained for $N$ points with time resolution $\Delta t$, the time $t=j \Delta t$, where $j$ is an integer. From the spatiotemporal discrete data $z_{i j}$, 2D Fourier-transformed discrete data $Z_{m k}$ are calculated by

$$
Z_{m k}=\frac{1}{M N} \sum_{i=0}^{M-1} \sum_{j=0}^{N-1} z_{i j} \exp \left[2 \pi \mathrm{I}\left(\frac{i m}{M}-\frac{j k}{N}\right)\right],
$$

where $I$ is the imaginary unit, $m$ is the poloidal mode number and the frequency $f=k \Delta f=k /(N \Delta t)$. The power spectrum $S_{m k}$ is calculated from $Z_{m k}$ by $S_{m k}=\left|Z_{m k}\right|^{2} / \Delta f$.

\section{BI-SPECTRAL ANALYSIS}

Bi-spectral analysis is executed for three waves (1,2 and 3) satisfying the matching condition $m_{1}+m_{2}=m_{3}$ and $f_{1}+f_{2}=f_{3}$. Bi-spectrum $B$ is defined as $B=\left\langle Z_{1} Z_{2} Z_{3}^{*}\right\rangle$. From the bi-spectrum, bi-coherence $b$ and bi-phase $\phi_{b}$ is calculated by

$$
b^{2}=\frac{|B|^{2}}{\left\langle\left|Z_{1} Z_{2}\right|^{2}\right\rangle\left\langle\left|Z_{3}\right|^{2}\right\rangle}, \phi_{b}=\tan ^{-1} \frac{\operatorname{Im} B}{\operatorname{Re} B} .
$$

Bi-coherence $b$ is in a region $0 \leq b \leq 1$, and indicates the degree of nonlinear coupling between the three waves. Bi-phase $\phi_{b}$ is in a region $-\pi \leq \phi_{b} \leq \pi$, and indicates the relationship of the phases between the three waves.

Received 26 September 2007; accepted 18 June 2008; published 27 July 2008.

\section{References}

1. Kadomtsev, B. B. Plasma Turbulence (Academic, New York, 1965).

2. Itoh, K., Itoh, S.-I. \& Fukuyama, A. Transport and Structural Formation in Plasmas (IOP Publishing, Bristol, 1999).

3. Diamond, P. H., Itoh, S.-I., Itoh, K. \& Hahm, T. S. Zonal flows in plasma-a review. Plasma Phys. Control. Fusion 47, R35-R161 (2005).

4. Schröder, C. et al. Mode selective control of drift wave turbulence. Phys. Rev. Lett. 86, $5711-5714$ (2001).

5. Inutake, M. Control of radial electric field and low-frequency fluctuations in open-ended systems. J. Plasma Fusion Res. SERIES 4, 75-81 (2001).

6. Kaneko, T., Tsunoyama, H. \& Hatakeyama, R. Drift-wave instability excited by field-aligned ion flow velocity shear in the absence of electron current. Phys. Rev. Lett. 90, 125001 (2003).

7. Sokolov, V \& Sen, A. K. New paradigm for the isotope scaling of plasma transport paradox. Phys. Rev. Lett. 92, $165002(2004)$

8. Schröder, C., Grulke, O., Klinger, T. \& Naulin, V. Drift waves in a high-density cylindrical helicon discharge. Phys. Plasmas 12, 042103 (2005).

9. Tynan, G. R. et al. Observation of turbulent-driven shear flow in a cylindrical laboratory plasma device. Plasma Phys. Control. Fusion 48, S51-S73 (2006).

10. Brochard, F., Bonhomme, G., Gravier, E., Oldenbürger, S. \& Philipp, M. Spatiotemporal control and synchronization of flute modes and drift waves in a magnetized plasma column. Phys. Plasmas 13, 052509 (2006).

11. Kim, Y. \& Powers, E. Digital bispectral analysis and its applications to nonlinear wave interactions. IEEE Trans. Plasma Sci. PS-7, 120-131 (1979).

12. Petviashvili, V. I. Self-focusing of an electrostatic drift wave. Sov. J. Plasma Phys. 3, 150-151 (1977).

13. Nozaki, K., Taniuti, T. \& Watanabe, K. Solitons in a convective motion of a low- $\beta$ plasma. II. J. Phys. Soc. Jpn. 46, 991-1001 (1979).

14. Drake, J. F., Zeiler, A. \& Biskamp, D. Nonlinear self-sustained drift-wave turbulence. Phys. Rev. Lett. 75, 4222-4225 (1995).

15. Champeaux, S. \& Diamond, P. H. Streamer and zonal flow generation from envelope modulations in drift wave turbulence. Phys. Lett. A 288, 214-219 (2001).

16. Shinohara, S. et al. Proc. 28th Int. Conf. on Phenomena in Ionized Gases, Prague, Czech Republic, 1P04-08, 354-357 (Institute of Plasma Physics, AS CR, Prague, 2007).

17. Terasaka, K. et al. Study on low-frequency fluctuations using new azimuthal probe array in high-density helicon linear plasma. Plasma Fusion Res. 2, 031 (2007).

18. Kasuya, N., Yagi, M., Itoh, K. \& Itoh, S.-I. Selective formation of turbulent structures in magnetized cylindrical plasmas. Phys. Plasmas 15, 052302 (2008). 
19. Yamada, T. et al. Fine positioning of a probe array. Rev. Sci. Instrum. 78, 123501 (2007).

20. Ritz, Ch. P., Powers, E. J. \& Bengtson, R. D. Experimental measurement of three-wave coupling and energy cascading. Phys. Fluids B 1, 153-163 (1989).

21. Xia, H. \& Shats, M. G. Inverse energy cascade correlated with turbulent-structure generation in toroidal plasma. Phys. Rev. Lett. 91, 155001 (2003).

22. Diamond, P. H. et al. Theory of two-point correlation for trapped electrons and the spectrum of drift wave turbulence. Plasma Phys. Control. Nuclear Fusion Res. Vol. 1, 259-269 (IAEA, Vienna, 1983).

\section{Acknowledgements}

We are grateful to P. H. Diamond, A. Fukuyama and G. R. Tynan for useful discussions, and F. Greiner and M. Kawaguchi for support in the experimental work. This work was supported by Grant-in-Aid for Specially-Promoted Research of MEXT, Japan (16002005) (Itoh project), Grant-in-Aid for Young Scientists (B) of JSPS, Japan (19760597), the collaboration programmes of the National Institute for Fusion Science (NIFS07KOAP017) and RIAM, Kyushu University and Asada Eiichi Research Foundation.

\section{Author contributions}

T.Y., T.M., Y.N., S.S., K.T., S.I. and Y.K. carried out the experiments. S.I.I. planned the research. N.K. and M.Y. carried out the simulations. T.Y., A.F. and K.I. analysed the data.

\section{Author information}

Reprints and permission information is available online at http://npg.nature.com/reprintsandpermissions. Correspondence and requests for materials should be addressed to T.Y. 